\title{
Aortic Valve Calcification as a Predictor of Post-Transcatheter Aortic Valve Replacement Pacemaker Dependence
}

\author{
Esseim Sharma a, b, h, Brian McCauleyc, Dhairyasheel S. Ghosalkar ${ }^{\mathrm{d}}$, Michael Atalaye, \\ Scott Collins ${ }^{\mathrm{f}}$, Anshul Parulkar ${ }^{\mathrm{d}}$, Wasiq Sheikh ${ }^{\mathrm{d}}$, Malik B. Ahmed ${ }^{\mathrm{d}}$, Antony Chu ${ }^{\mathrm{g}}$
}

\begin{abstract}
Background: Atrioventricular block requiring permanent pacemaker (PPM) implantation is a common complication of transcatheter aortic valve replacement (TAVR). The mechanism of atrioventricular (AV) block during TAVR is not fully understood, but it may be due to the mechanical stress of TAVR deployment, resulting in possible injury to the nearby compact AV node. Aortic valve calcification (AVC) may worsen this condition and has been associated with an increased risk for post-TAVR PPM implantation. We performed a retrospective analysis to determine if AVC is predictive for long-term right ventricular (RV) pacing in post-TAVR pacemaker patients at 30 days.
\end{abstract}

Methods: A total of 262 consecutive patients who underwent TAVR with a balloon-expandable valve were analyzed. AVC data were derived from contrast-enhanced computed tomography and characterized by leaflet sector and region.

Results: A total of 25 patients (11.1\%) required post-TAVR PPM implantation. Seventeen patients did not require RV pacing at 30 days. Nine of these 17 patients had no RV pacing requirement within 10 days. The presence of intra-procedural heart block $(\mathrm{P}=0.004)$ was the only significant difference between patients who did not require PPM and those who required PPM but they were not RV pacing-dependent at 30 days. Non-coronary cusp (NCC) calcium volume was significantly higher in patients who were pacemaker-dependent at 30

Manuscript submitted January 4, 2020, accepted January 23, 2020

aDepartment of Cardiology, The Warren Alpert Medical School of Brown University, Providence, RI, USA

bDepartment of Cardiology, Rhode Island Hospital, Providence, RI, USA

'Department of Cardiology, University of Pennsylvania, Philadelphia, PA, USA

${ }^{\mathrm{d} D e p a r t m e n t}$ of Internal Medicine, The Warren Alpert Medical School of Brown University, Providence, RI, USA

eDepartment of Diagnostic Imaging and Medicine, The Warren Alpert Medical School of Brown University, Providence, RI, USA

fDepartment of Diagnostic Imaging and Medicine, Rhode Island Hospital, Providence, RI, USA

gDepartment of Medicine, The Warren Alpert Medical School of Brown University, Providence, RI, USA

${ }^{\mathrm{h}}$ Corresponding Author: Esseim Sharma, Department of Cardiology, Rhode Island Hospital, 593 Eddy Street, Providence, RI 02903, USA.

Email: esseim.sharma@lifespan.org

doi: https://doi.org/10.14740/cr1011 days $(\mathrm{P}=0.01)$ and a calcium volume of $>239.2 \mathrm{~mm}^{3}$ in the NCC was strongly predictive of pacemaker dependence at 30 days (area under the curve $(\mathrm{AUC})=0.813)$. Pre-existing right bundle branch block (RBBB) (odds ratio (OR) 105.4, $\mathrm{P}=0.004$ ), bifascicular block (OR 12.5, $\mathrm{P}=0.02)$, QRS duration $(\mathrm{OR} 70.43, \mathrm{P}=0.007)$ and intraprocedural complete heart block (OR 12.83, $\mathrm{P}=0.03$ ) were also predictive of pacemaker dependence at 30 days.

Conclusions: In patients who required PPM after TAVR, quantification of AVC by non-coronary leaflet calcium volume was found to be a novel predictor for RV pacing dependence at 30 days. The association of NCC calcification and PPM dependence may be related to the proximity of the conduction bundle to the non-coronary leaflet. Further studies are necessary to improve risk prediction for long-term RV pacing requirements following TAVR.

Keywords: Pacing; Electrophysiological; Clinical; Imaging; Noninvasive risk assessment tests; Transcatheter aortic valve replacement

\section{Introduction}

The development of atrioventricular (AV) block requiring permanent pacemaker (PPM) implantation is a well-known complication of transcatheter aortic valve replacement (TAVR) [1-4]. Rates of PPM implantation after TAVR are variably reported in the literature and are influenced by multiple factors, including the type of valve used as well as procedural and patient-specific risk factors $[5,6]$. Aortic valve calcification as quantified on pre-TAVR computed tomography (CT) scans has been proposed as a novel and highly predictive risk factor of PPM implantation after TAVR [7].

The mechanism of conduction disturbances following TAVR is incompletely understood, contributing to the difficulty in predicting which patients will require PPM implantation after the procedure. The mechanical stress of deployment and resultant tissue edema are thought to be possible contributors, resulting in possible injury to the nearby AV node and left bundle branch $[8,9]$. Multiple studies have reported eventual recovery of intrinsic conduction in a significant portion of patients who received PPM after TAVR, sometimes as early as 10 days post-implantation [10-13]. Because the decision to move forward with PPM implantation after TAVR can have significant clinical and economic ramifications, identifying the 
possibility of conduction system recovery after TAVR would be helpful in guiding management strategies regarding pacemaker implantation [14-16].

Though many studies have examined risk factors for PPM implantation after TAVR, few have looked at risk factors for pacemaker dependence after the procedure, especially within the first 30 days post-procedure. In this study, the utility of aortic valve calcification as quantified on CT scan as a predictor for the need for right ventricular (RV) pacing after TAVR is examined.

\section{Materials and Methods}

\section{Study population}

The study population consisted of 262 consecutive patients who underwent TAVR at a single center from March 2012 to October 2016. Prior to TAVR, all patients underwent coronary angiography, computed tomographic angiography of the chest, abdomen, and pelvis, and 2D echocardiography. A multidisciplinary team consisting of at least two cardiothoracic surgeons and an interventional cardiologist then evaluated the patients and accepted them for TAVR. All patients received a balloonexpandable Edwards SAPIEN valve (Edwards Life Sciences, Inc.). Three different generations of the SAPIEN valves were used in this study: SAPIEN, SAPIEN XT and SAPIEN III (Table 1). Thirty-six patients with PPM implantation prior to the TAVR were excluded from the study cohort. No patients met guideline indications for permanent pacing prior to TAVR.

\section{Patient characteristics}

Baseline demographic, electrocardiographic, procedural and imaging data were collected on each patient as part of the routine pre-TAVR workup. All electrocardiography (ECG) data were obtained based on pre-operative 12-lead ECGs obtained during the index admission within $24 \mathrm{~h}$ prior to TAVR. Data regarding intra-procedural parameters were obtained from the procedure notes.

\section{Pacemaker and interrogation data}

All patients who received PPM implants met guideline indications for post-procedural permanent pacing at the time of implantation, as determined by a consulting board-certified electrophysiologist. Patients did not receive an EP study prior to a decision being made regarding PPM implantation. The most common indication for pacemaker implantation was complete heart block (CHB) (Fig. 1). These patients had cardiology follow-up within 10 days and an additional follow-up within 1 month after implantation. Pacing and underlying rhythm data were obtained during device interrogations at these visits. Patients were considered pacemaker-dependent if their spontaneous ventricular rate was less than 30 beats/min during backup pacing set at 30 beats/min for $30 \mathrm{~s}$, as done in prior studies $[10,17]$.

\section{Multidetector computed tomography (MDCT) image ac- quisition}

An electrocardiographically gated multidetector CT study was performed only if the patient's renal function was considered satisfactory, as is routine clinical practice; this was generally when the serum creatinine was $\leq 2.0 \mathrm{mg} / \mathrm{dL}$. Patients were evaluated using a Siemens Somatom Cardiac 64 or Siemens Somatom Flash scanner (Siemens Medical Solutions USA, Inc., Malvern, PA) using collimation of $0.6 \mathrm{~mm}$ at a fixed pitch of 0.2 with an injection of 50 to $110 \mathrm{~mL}$ of iopamidol (Isovue-370). A dedicated protocol was formulated, with $120 \mathrm{kV}$ and tube current modified according to the patient's size. Image acquisition was, for the most part, performed with retrospective electrocardiographic gating. CT Digital Imaging and Communications in Medicine (DICOM) data were analyzed by a dedicated advanced imaging core laboratory using TeraRecon Aquarius software (San Mateo, CA).

\section{Analysis of aortic valve complex calcification}

The protocol for analysis of aortic valve complex calcification used in this study was modified from existing published reports $[7,18]$. The aortic annulus was defined as the virtual plane that contained the basal points of attachment of all three aortic valve leaflets in the proximal left ventricular outflow tract (LVOT). The late systolic phase (35\% of the R-R interval) was used during image analysis for best image quality. Calcium scoring was reported in calcium volume rather than Agatston units, in keeping with prior studies in the field and in light of concerns regarding reproducibility of volume scoring [19-22].

A cutoff of 550 Hounsfield Units (HU) was used to quantify areas of calcium within the aortic valve calcification. However, given the variability in luminal enhancement inherent to contrast-induced images, in patients with a luminal attenuation $<200 \mathrm{HU}$, a cutoff of $300 \mathrm{HU}$ was used. For patients with luminal attenuation $>500 \mathrm{HU}$, a cutoff of $50 \mathrm{HU}$ greater than the luminal attenuation was used [18].

To analyze the aortic valve complex, the virtual basal ring was created using the double-oblique method by identifying the insertion of each of the aortic cusps at the annular plane. This technique is commonly used to size the annulus prior to TAVR procedures [23]. The process of how the virtual basal ring was created is depicted and described in Figure 2a-d. The aortic valve complex was then batched and separated along the craniocaudal axis of the LVOT/aortic valve (Fig. 3a) to exclude calcification from neighboring regions such as the aortomitral continuity. The region of interest spanned from $5 \mathrm{~mm}$ below the annular plane to the superior edge of the leaflets. Areas of calcification could then be individually traced by the reader and scored separately as needed after adjusting for the luminal attenuation (Fig. 3b-d).

The following regions of the aortic valve complex were scored: the LVOT region (from $5 \mathrm{~mm}$ below the annular plane to the annular plane), the device-landing zone (DLZ) $(2 \mathrm{~mm}$ 
Table 1. Electrocardiographic Data, Clinical Imaging Data and Procedural Parameters of Non-RV Pacing-Dependent and RV Pacing-Dependent Patients at 1 Month Post-PPM Implant

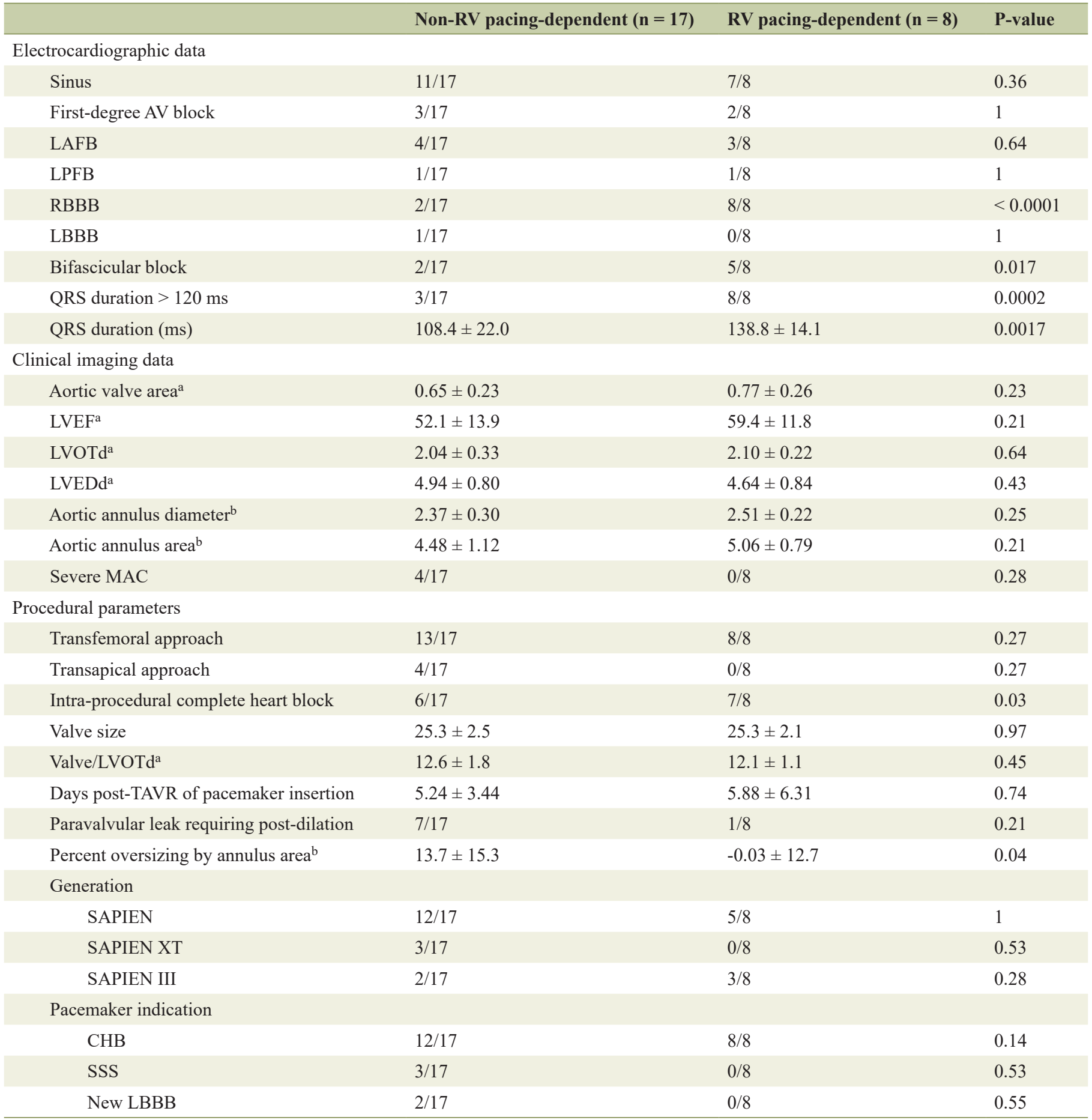

The P-values shown are derived from two-tailed Fisher's exact test for categorical variables and two-tailed unpaired Student's $t$-test for continuous variables. Patients who were non-RV pacing-dependent at 30 days and patients who were RV pacing-dependent at 30 days patients were separately compared with the non-pacemaker population to derive these P-values. Continuous variables are reported as the mean plus or minus the standard

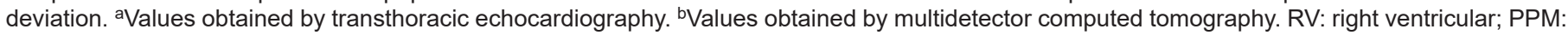
permanent pacemaker; AV: atrioventricular; LAFB: left anterior fascicular block; LPFB: left posterior fascicular block; RBBB: right bundle branch block; LBBB: left bundle branch block; LVEF: left ventricular ejection fraction; LVEDd: left ventricular end diastolic diameter; LVOTd: left ventricular outflow tract diameter; MAC: mitral annular calcification; TAVR: transcatheter aortic valve replacement; CHB: complete heart block; SSS: sick sinus syndrome; LVOT: left ventricular outflow tract. 


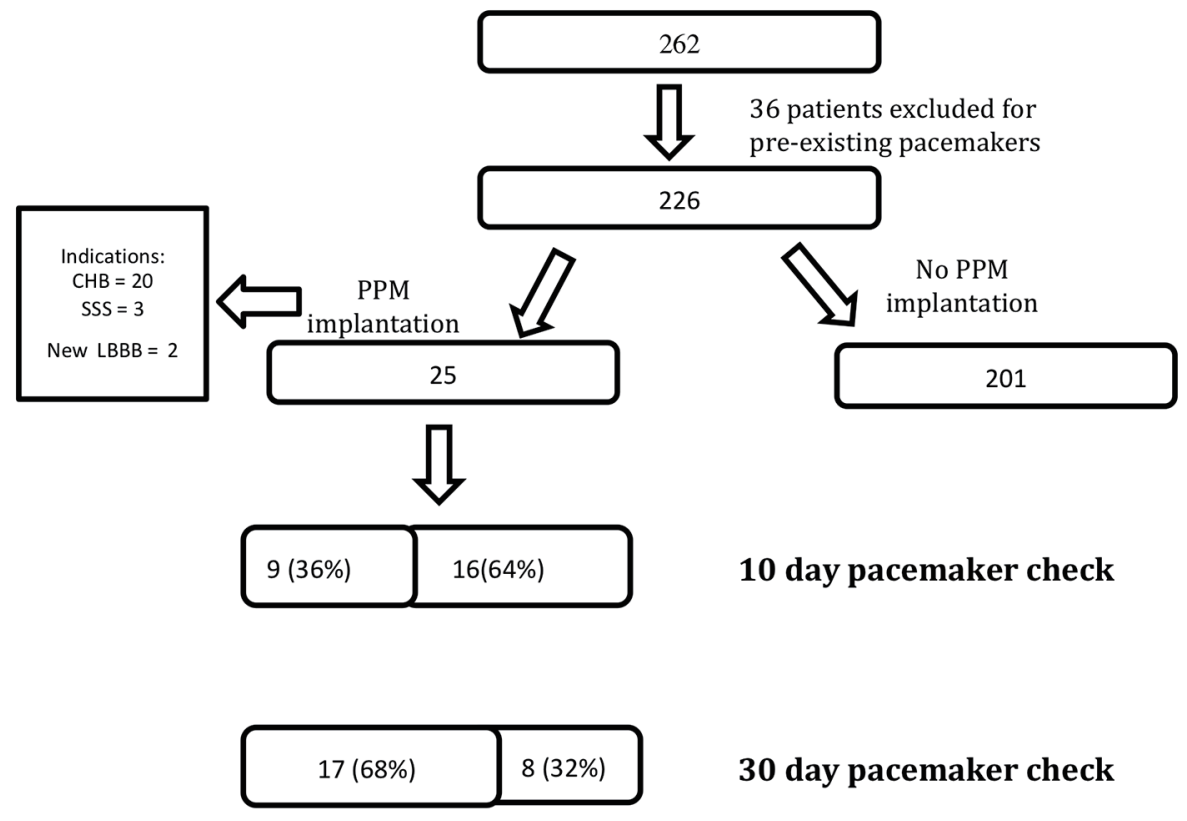

Figure 1. A total of 262 patients underwent transcatheter aortic valve replacement (TAVR), 36 patients were excluded from analysis because of pre-existing pacemakers and 25 patients required pacemaker implantation after TAVR for three different indications. All patients received a pacemaker check at 10 and 30 days. At 10 days, nine patients were no longer right ventricular (RV) pacingdependent. At 30 days, 17 patients were no longer RV pacing-dependent. CHB: complete heart block; SSS: sick sinus syndrome; LBBB: left bundle branch block; PPM: permanent pacemaker.

a

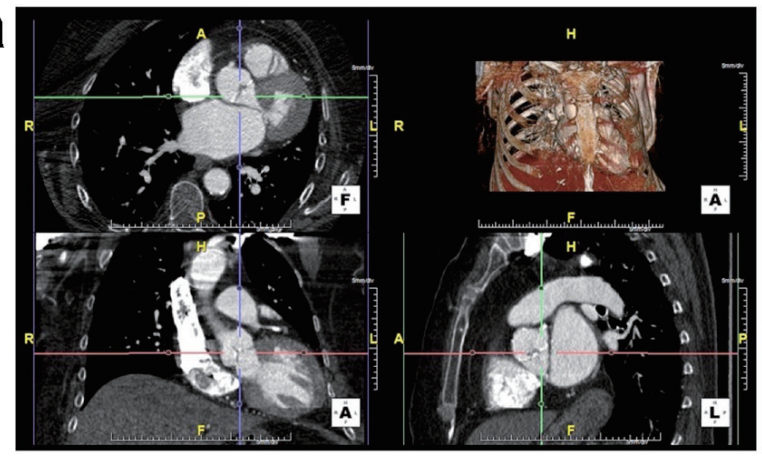

C

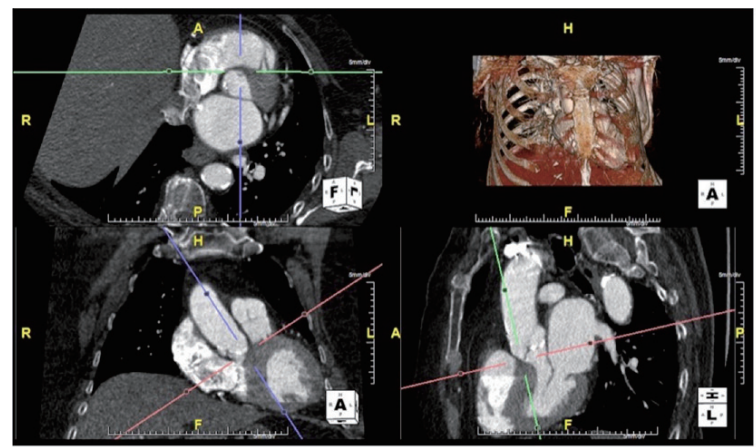

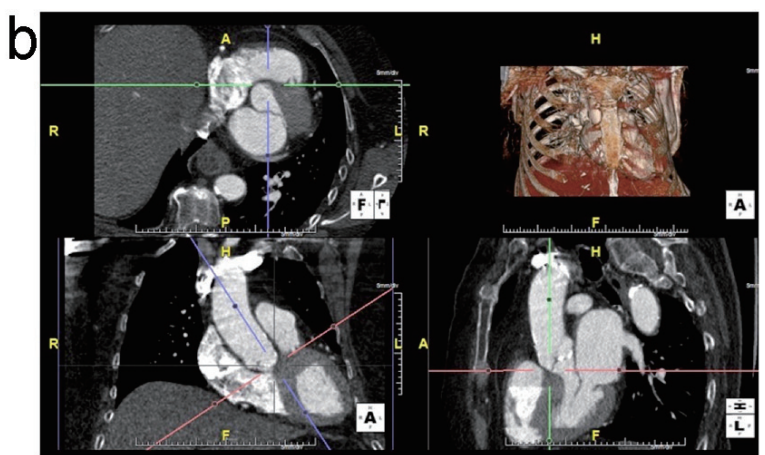

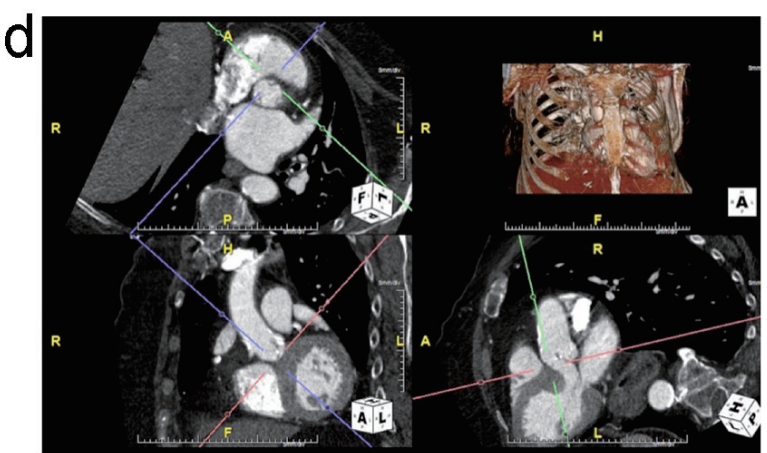

Figure 2. (a) Unaligned view of the aortic annulus that is not in plane. The top-left is the axial view, bottom-left is the transverse view and the bottom-right is the sagittal view. (b) The right coronary cusp (RCC) is now visible in the axial view by adjusting the red axes. The green and purple axes are then centered on the insertion of the RCC. (c) The non-coronary cusp (NCC) insertion is aligned by adjusting the red axes in the sagittal image to bring it into plane with the RCC insertion in the axial view. (d) The purple axes are adjusted to run through both RCC and NCC insertions. Then, the red axes on the transverse view are adjusted to bring in the insertion of the left coronary cusp (LCC) into the same plane as the RCC and NCC. 

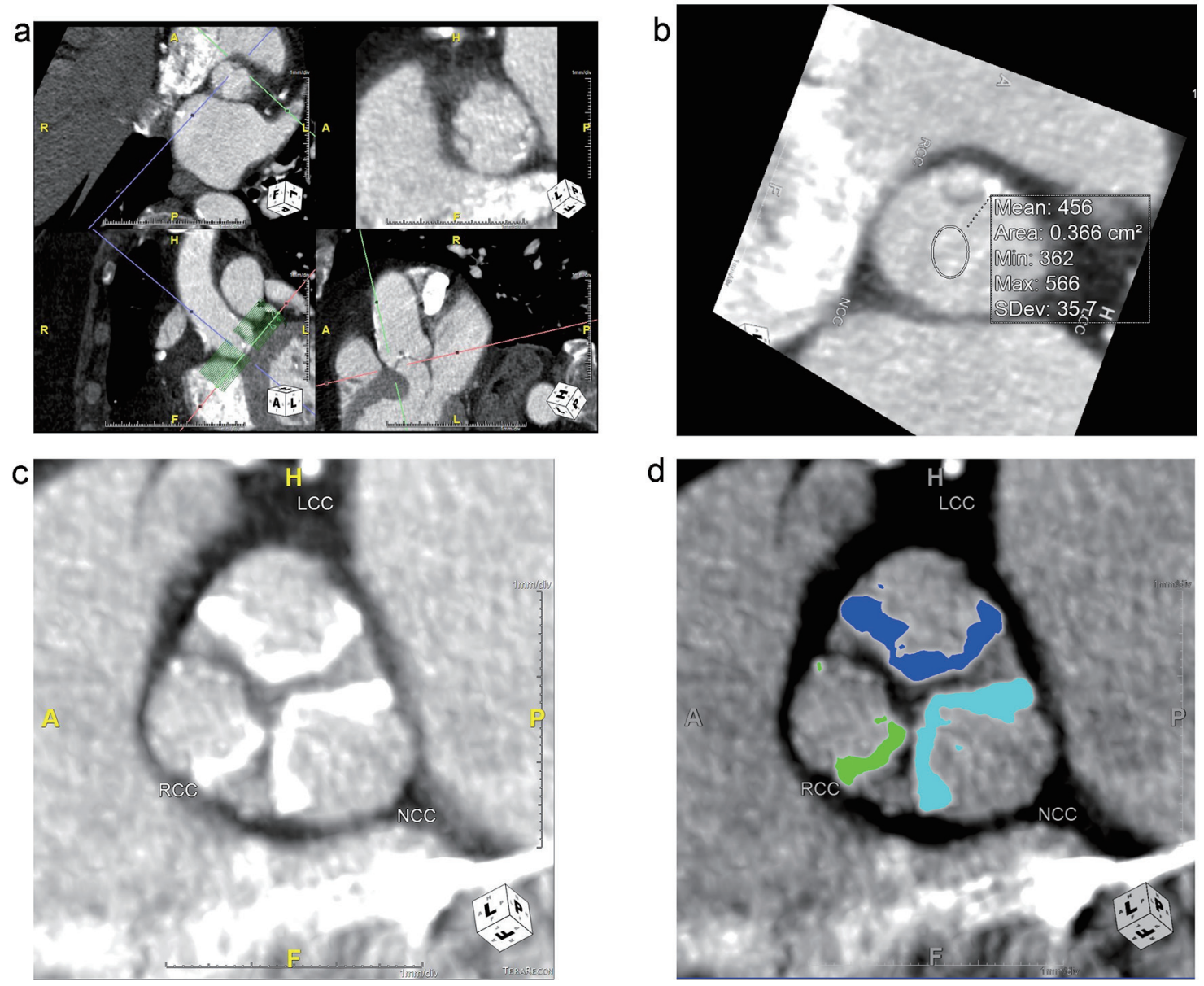

Figure 3. (a) The aortic valve complex used for analysis is created using a batching tool to separate the study images from the larger, original. The batching tool is visible in the bottom left in the axial view as a box of green lines, and it is centered at the annular plane (red axes). It extends $5 \mathrm{~mm}$ caudally from the annular plane and cranially to the tip of the upper leaflets. Each green line represents $1 \mathrm{~mm}$. The output at the level of the annular plane is in the top-right box, with the cusps labeled as right coronary cusp (RCC), left coronary cusp (LCC) and non-coronary cusp (NCC) for orientation. (b) Tool used to calculate mean attenuation in Hounsfield Units (HU) to adjust cutoffs for calcium scoring. In this image, the mean luminal attenuation is $456 \mathrm{HU}$, so a cutoff of $550 \mathrm{HU}$ was used. (c) Zoomed in view of leaflet calcification in one slice of the upper leaflet zone for each leaflet. (d) Calcification of each leaflet in this slice in the upper leaflet zone was traced using calcium analysis software, which then provided a calcium score, volume and mean. In the device landing zone and annulus region, a total calcium score, volume and mean was provided.

inferior to the annular plane to $3 \mathrm{~mm}$ superior to the annular plane) and the upper leaflet region (3 $\mathrm{mm}$ superior to the annular plan to the superior edge of the leaflets) (Fig. 4). Each leaflet was separately quantified in the upper leaflet region (Fig. 3d).

\section{Statistics}

Data were retrospectively analyzed. All categorical variables were analyzed between the two groups using the two-tailed Fisher's exact test. Data are reported as numbers and percentages. Continuous variables were analyzed using the two-tailed unpaired Student's $t$-test and are reported as mean values and the standard deviation. Statistical analysis was performed using the STATA SE 14 Software (Stata Corp LP, College Station, TX, USA).

\section{Ethical compliance with human study}

The study protocol was approved by the local Institutional Review Board and adhered to the principles of the Declaration of Helsinki.

\section{Results}

\section{Baseline characteristics}

The baseline characteristics of this study population, encompassing demographic, electrocardiographic, procedural and imaging data are presented in Table 2. Patients who did not require PPM implantation after TAVR are compared with patients who required PPM implantation after TAVR but 

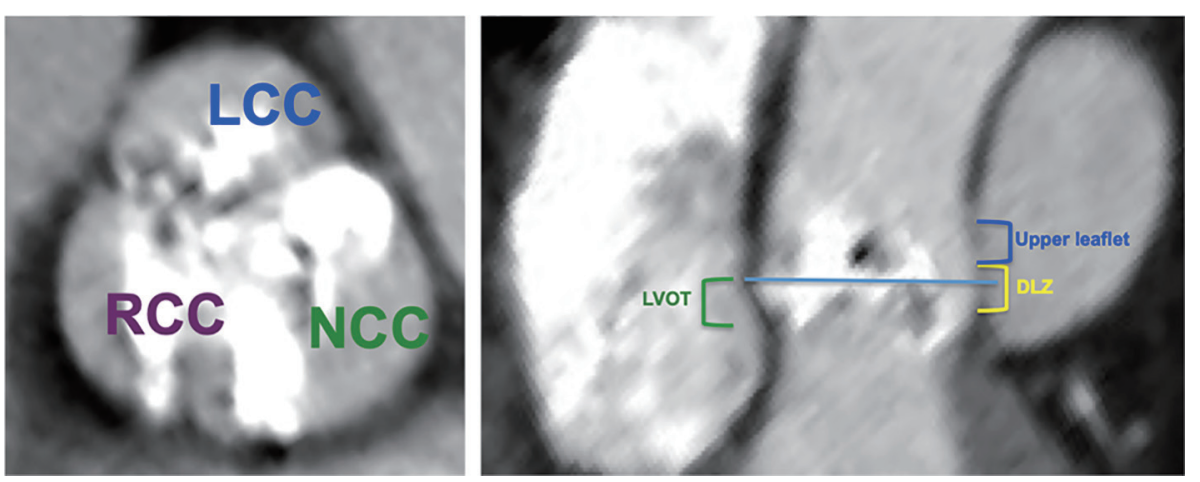

Figure 4. The aortic valve complex was separated in the craniocaudal axis of the left ventricular outflow tract (LVOT)/aortic valve into the following regions: the LVOT region (from $5 \mathrm{~mm}$ below the annular plane to the annular plane, green), the device-landing zone ( $2 \mathrm{~mm}$ inferior to the annular plane to $3 \mathrm{~mm}$ superior to the annular plane, yellow) and the upper leaflet region ( $3 \mathrm{~mm}$ superior to the annular plan to the superior edge of the leaflets, blue). Each leaflet was separately quantified in the upper leaflet region. NCC: non-coronary cusp; LCC: left coronary cusp; RCC: right coronary cusp.

were not pacemaker-dependent at 30 days. There were no significant differences between these two groups except for a higher rate of intra-procedural CHB in the PPM implantation group $(\mathrm{P}=0.004)$. Table 2 also compares patients who did not require PPM implantation after TAVR with patients who required PPM implantation after TAVR and were pacemaker-dependent at 30 days. One patient who did not receive a PPM post-TAVR did not have any electrocardiographic data. Patient who were pacemaker-dependent at 30 days had a significantly higher estimated glomerular filtration rate (eGFR) $<60(\mathrm{P}=0.035)$, higher baseline creatinine $(\mathrm{P}=0.0002)$ and lower rate of hypertension than patients who did not require PPM implantation. Additionally, there was a significantly higher amount of pre-existing right bundle branch block (RBBB) $(\mathrm{P}<0.001)$, bifascicular block $(\mathrm{P}<0.001)$, QRS duration $(\mathrm{P}=0.0002)$ and intra-procedural heart block $(\mathrm{P}<$ $0.001)$.

Table 3 compares the baseline demographic and clinical characteristics between patients who were pacemaker-dependent at 30 days and patients who were not pacemaker-dependent at 30 days. There was no significant difference in baseline characteristics between these two groups.

\section{Indications for PPM implantation}

A total of 25 patients underwent PPM implantation after TAVR in this study. The most common indication for PPM implantation was CHB (80\%), followed by sick sinus syndrome (SSS, $12 \%$ ) and new left bundle branch block (LBBB, 8\%). All patients who were pacemaker-dependent at 30 days post-TAVR received PPM implants for CHB. There was no significant difference between indications for PPM implantation between the two groups (Table 1).

\section{Predictors of $\mathrm{RV}$ pacing requirements at 30 days after PPM implant}

A comparison of electrocardiographic, imaging and procedural parameters between patients who were pacemaker-dependent at 30 days after PPM implant and those who were not pacemaker-dependent at 30 days after PPM implant are reported in Supplementary Material 1 (www.cardiologyres.org). Patients who were pacemaker-dependent at 30 days had significantly higher rates of RBBB $(\mathrm{P} \leq 0.0001)$, bifascicular block $(\mathrm{P}=$ $0.017)$, $\mathrm{QRS}$ duration $>120 \mathrm{~ms}(\mathrm{P}=0.002)$ and intra-procedural CHB $(P=0.03)$ than patients who were not pacemakerdependent at 30 days. There was also a significant difference in $\mathrm{QRS}$ duration as a continuous variable $(\mathrm{P}=0.0017)$. There were no echocardiographic parameters that were significantly different between the two groups (Table 1). Patients who were pacemaker-dependent at 30 days had significantly less oversizing of their valves than patients who were not pacemaker-dependent at 30 days $(P=0.04)$. There was no significant difference in the type or generation of valve between the two groups (Table 1).

Aortic valve calcification data for patients who were pacemaker-dependent at 30 days compared with patients who were not pacemaker-dependent at 30 days are shown in Table 4. There was no significant difference in mean attenuation $(\mathrm{P}=$ $0.72)$ or Hounsfield Units (HU) cutoff $(P=0.62)$ between the two groups. One patient who was not pacemaker-dependent at 30 days was excluded from analysis because of a pre-existing prosthetic aortic valve (valve-in-valve), which interfered with calcium quantification. Compared with patients who were not pacemaker-dependent at 30 days, patients who were pacemaker-dependent at 30 days had a significantly higher non-coronary cusp (NCC) leaflet calcium volume $(\mathrm{P}=0.006)$. There was also a trend towards higher total calcium volume in the total upper leaflet zone $(\mathrm{P}=0.07)$ in patients who were pacemaker-dependent at 30 days.

The distribution of calcium volume in the leaflet region was similar between the two groups, with about half of the leaflet calcium located in the NCC. Calcium distribution in the leaflet zone was not significantly associated with pacemaker dependence at 30 days (Supplementary Material 1, www.cardiologyres.org).

All patients who were pacemaker-dependent at 30 days post-implant had pre-existing RBBBs, while only two out 
Table 2. Baseline Characteristics and Comparison of Patients Not Requiring PPM Implant and Those Requiring PPM Implant PostTAVR

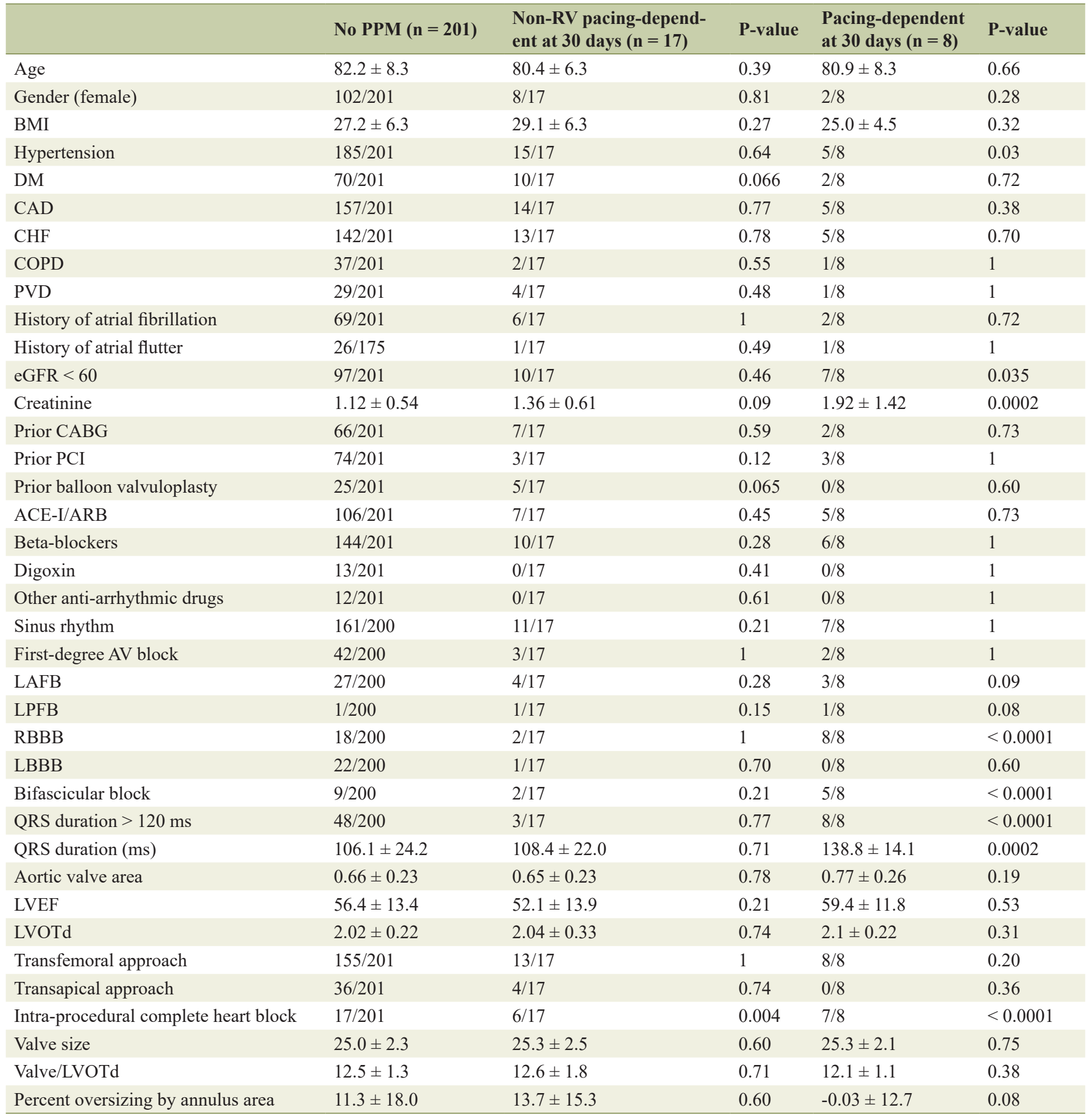

The P-values shown are derived from two-tailed Fisher's exact test for categorical variables and two-tailed unpaired Student's $t$-test for continuous variables. Patients who were non-RV pacing-dependent at 30 days and patients who were RV pacing-dependent at 30 days patients were separately compared with the non-pacemaker population to derive these P-values. Continuous variables are reported as the mean plus or minus the standard deviation. PPM: permanent pacemaker; TAVR: transcatheter aortic valve replacement; RV: right ventricular; BMI: body mass index; DM: diabetes mellitus; CAD: coronary artery disease; CHF: congestive heart failure; COPD: chronic obstructive pulmonary disease; PVD: peripheral vascular disease; eGFR: estimated glomerular filtration rate; CABG: coronary artery bypass grafting; $\mathrm{PCl}$ : percutaneous coronary intervention; $\mathrm{ACE}-\mathrm{I} / \mathrm{ARB}$ : angiotensin converting enzyme inhibitor/angiotensin receptor blocker; AV: atrioventricular; LAFB: left anterior fascicular block; LPFB: left posterior fascicular block; RBBB: right bundle branch block; LBBB: left bundle branch block; LVEF: left ventricular ejection fraction; LVOTd: left ventricular outflow tract diameter. 
Table 3. Baseline Demographic and Clinical Characteristics of Non-RV Pacing-Dependent and Pacing-Dependent Patients at 1 Month Post-PPM Implant

\begin{tabular}{|c|c|c|c|}
\hline & Non-RV pacing-dependent $(n=17)$ & RV pacing-dependent $(n=8)$ & P-value \\
\hline Age & $80.4 \pm 6.3$ & $80.9 \pm 8.3$ & 0.88 \\
\hline Gender (female) & $8 / 17$ & $2 / 8$ & 0.40 \\
\hline Hypertension & $15 / 17$ & $5 / 8$ & 0.28 \\
\hline $\mathrm{DM}$ & $10 / 17$ & $2 / 8$ & 0.2 \\
\hline $\mathrm{CHF}$ & $13 / 17$ & $5 / 8$ & 0.64 \\
\hline COPD & $2 / 17$ & $1 / 8$ & 1 \\
\hline PVD & $4 / 17$ & $1 / 8$ & 0.64 \\
\hline History of atrial fibrillation & $6 / 17$ & $2 / 8$ & 0.68 \\
\hline History of atrial flutter & $1 / 17$ & $1 / 8$ & 1 \\
\hline Prior PCI & $3 / 17$ & $3 / 8$ & 0.34 \\
\hline Prior balloon valvuloplasty & $5 / 17$ & $0 / 8$ & 0.14 \\
\hline ACE-I/ARB & $7 / 17$ & $5 / 8$ & 0.41 \\
\hline Beta-blockers & $10 / 17$ & $6 / 8$ & 0.66 \\
\hline Digoxin & $0 / 17$ & $0 / 8$ & 1 \\
\hline Other anti-arrhythmic drugs & $0 / 17$ & $0 / 8$ & 1 \\
\hline
\end{tabular}

The P-values shown are derived from two-tailed Fisher's exact test for categorical variables and two-tailed unpaired Student's $t$-test for continuous variables. Patients who were non-RV pacing-dependent at 30 days and patients who were RV pacing-dependent at 30 days patients were separately compared with the non-pacemaker population to derive these P-values. Continuous variables are reported as the mean plus or minus the standard deviation. RV: right ventricular; PPM: permanent pacemaker; BMI: body mass index; DM: diabetes mellitus; CAD: coronary artery disease; CHF: congestive heart failure; COPD: chronic obstructive pulmonary disease; PVD: peripheral vascular disease; eGFR: estimated glomerular filtration rate; CABG: coronary artery bypass grafting; PCI: percutaneous coronary intervention; ACE-I/ARB: angiotensin converting enzyme inhibitor/angiotensin receptor blocker.

of 17 patients who were not pacemaker-dependent at 30 days post-implant had pre-existing RBBBs (odds ratio $(\mathrm{OR})$ $105.4,4.52-2458.5, \mathrm{P}=0.004)$. The presence of a pre-existing bifascicular block (OR 12.50, $1.6-97.65, \mathrm{P}=0.02), \mathrm{QRS}$ duration $>120 \mathrm{~ms}(\mathrm{OR} 70.43,3.23-1535.22, \mathrm{P}=0.007)$ and intra-procedural CHB (OR 12.83, 1.26 - 130.52, P = 0.03) were also strongly associated with pacemaker dependence at 30 days (Table 5). The positive predictive value of RBBB in determining pacemaker dependence pacing at 30 days was $88.2 \%$.

Figure 5 shows the receiver operating characteristic (ROC) curve for NCC calcium volume and pacemaker dependence at 30 days. Using a cutoff of $>239 \mathrm{~mm}^{3}$, calcium volume in the NCC leaflet was predictive of pacemaker dependence at 30 days $(\mathrm{AUC}=0.813)$.

\section{Pacemaker follow-up}

Twenty-five patients received pacemakers after TAVR in this study. Within 10 days of pacemaker implantation, there were nine patients (36\%) who were no longer pacemaker-dependent. At 30 days post-PPM implantation, 17 patients $(68 \%)$ were no longer pacemaker-dependent (Fig. 1). Non-paced surface ECGs for the 17 patients who were not pacemaker-dependent at 30-day follow-up showed recovery back to their pre-TAVR rhythm in all but one patient, who had developed new firstdegree AV block at follow-up.

\section{Patients with early recovery of cardiac conduction}

To further characterize the nine patients with evidence of early recovery of cardiac conduction, these nine patients were compared with the remaining eight patients who were no longer pacemaker-dependent at 30 days and the eight patients who remained pacemaker-dependent at 30 days. Pre-existing RBBB, pre-existing bifascicular block, pre-existing QRS duration $>$ $120 \mathrm{~ms}$ and intra-procedural CHB were all still significantly different between the non-RV pacing-dependent patients at 10 days when compared with the RV pacing-dependent patients at 10 days. There were no significant differences between de- 
Table 4. Aortic Valve Complex Calcification

\begin{tabular}{llll}
\hline & $\begin{array}{l}\text { RV pacing-dependent } \\
\text { at } 30 \text { days }(\mathbf{n = 8})\end{array}$ & $\begin{array}{l}\text { Non-RV pacing-dependent } \\
\text { at 30 days (n= 16) }\end{array}$ & $\begin{array}{c}\text { P-value } \\
\text { Mean attenuation }\end{array}$ \\
\hline $336.6 \pm 87.6$ & $353.8 \pm 120.2$ & 0.72 \\
\hline HU cutoff & $353.8 \pm 70.7$ & $539.8 \pm 66.9$ & 0.62 \\
\hline Total calcium volume & $783.5 \pm 622.1$ & $420.3 \pm 264.2$ & 0.055 \\
\hline LVOT calcium volume & $673.7 \pm 202.8$ & $697.6 \pm 34.7$ & 0.18 \\
\hline Annulus calcium volume & $117.9 \pm 120.6$ & $92.9 \pm 50.4$ & 0.48 \\
RCC leaflet calcium volume & $119.7 \pm 121.5$ & $107.2 \pm 119.9$ & 0.81 \\
\hline NCC leaflet calcium volume & $342.0 \pm 290.8$ & $113.6 \pm 63.2$ & 0.006 \\
\hline LCC leaflet calcium volume & $139.9 \pm 132.7$ & $91.4 \pm 87.0$ & 0.29 \\
Total leaflet calcium volume & $601.6 \pm 504.9$ & $312.2 \pm 248.8$ & 0.07 \\
\hline
\end{tabular}

The P-values shown are derived from two-tailed unpaired Student's $t$-test for continuous variables. Patients who were non-RV pacing-dependent at 30 days and patients who were RV pacing-dependent at 30 days patients were separately compared. Continuous variables are reported as the mean plus or minus the standard deviation. HU: Hounsfield Units; LVOT: left ventricular outflow tract; RCC: right coronary cusp; NCC: non-coronary cusp; LCC: left coronary cusp; RV: right ventricular.

mographic, electrocardiographic, imaging, or procedural parameters between the nine patients with early recovery of their conduction system at 10 days and the eight non-RV pacing-dependent patients with late recovery of their conduction system at 30 days (Supplementary Material 2, www.cardiologyres. org).

Calcium scoring was also compared among patients who had early recovery of their conduction, patients who had late recovery of their cardiac conduction and patients who did not have recovery of their cardiac conduction at all. One patient was excluded from the calcium scoring analysis, as in the prior analysis, because of a prior valve-in-valve procedure. There were no significant differences in calcium scoring between patients with early and late recovery of their conduction. However, both groups had a significantly lower NCC volume than patients with no recovery of their conduction system (Supplementary Material 3, www.cardiologyres.org).

\section{Discussion}

This single-center study of 262 consecutive patients who underwent TAVR with a balloon-expandable valve at a single in- stitution builds on our previous findings in describing predictors of persistent cardiac conduction abnormalities after TAVR and introduces aortic valve calcification as a novel predictor of pacemaker dependence. As described previously, conduction abnormalities after TAVR are often transient and resolve over time. Out of 25 patients $(9.5 \%)$ who received PPM implants, $36 \%$ of patients were no longer pacemaker-dependent 10 days after implantation and $68 \%$ of patients were no longer pacemaker-dependent at 30 days post-implantation. Electrocardiographic parameters such as RBBB, bifascicular block, QRS duration and intra-procedural CHB were predictive of pacemaker dependence at 30 days [13].

Aortic valve calcification in the NCC leaflet was found to be significantly associated with pacemaker dependence at 30 days. This novel predictor had a strong predictive value for pacemaker dependence at 30 days. Using a cutoff of $>239$ $\mathrm{mm}^{3}$, NCC leaflet calcium volume was strongly predictive of pacemaker dependence at 30 days post-implant, with an AUC of 0.813 . Distribution of aortic valve complex calcification was not associated with pacemaker dependence; rather, only the absolute calcium volume was predictive.

Calcification of the aortic valve complex by CT scan has been studied as a predictor for PPM implantation after TAVR.

Table 5. Predictors of Persistent RV Pacing After PPM Implantation

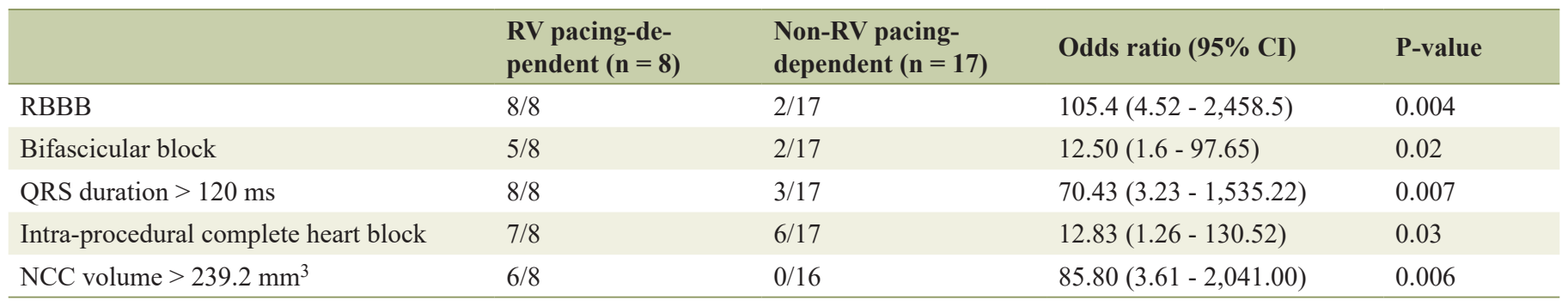

The P-values shown are derived from two-tailed Fisher's exact test for categorical variables. Patients who were non-RV pacing-dependent at 30 days and patients who were RV pacing-dependent at 30 days patients were separately compared. RBBB: right bundle branch block; Cl: confidence interval; NCC: non-coronary cusp; RV: right ventricular; PPM: permanent pacemaker. 


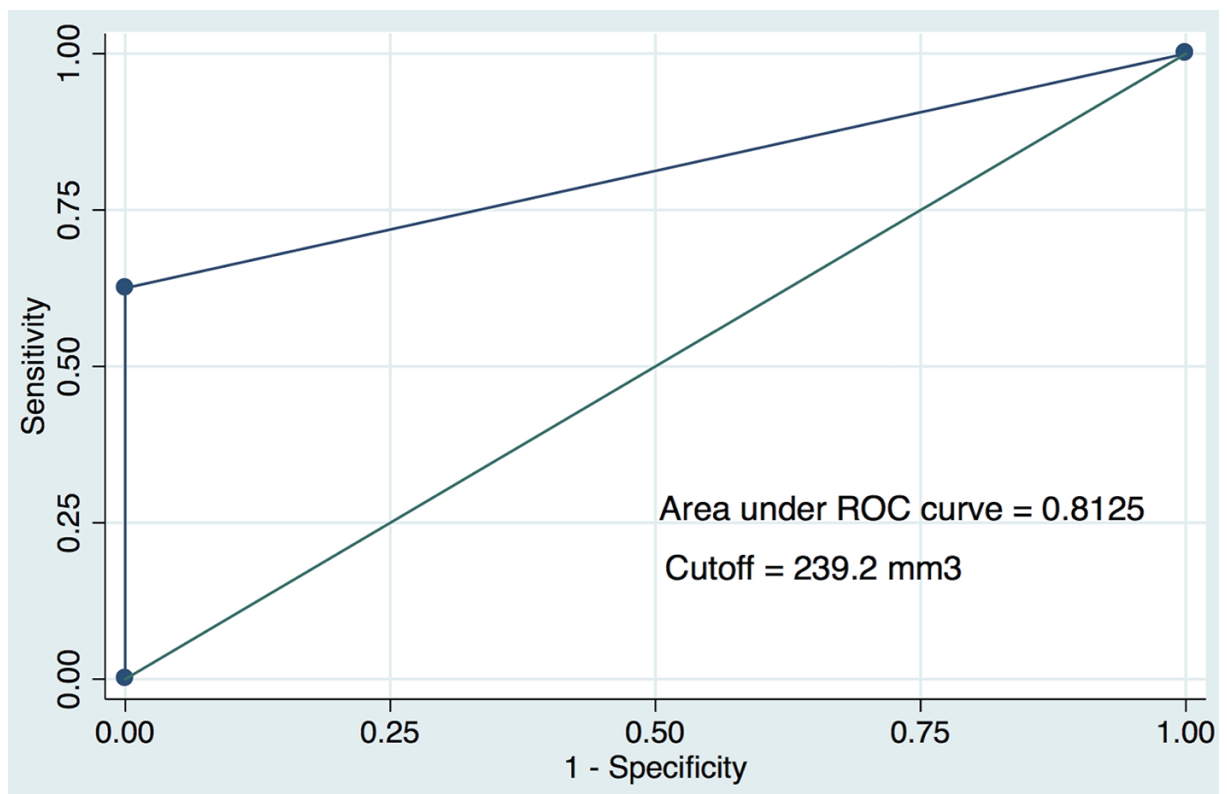

Figure 5. The receiver operating characteristic (ROC) curve for non-coronary cusp (NCC) volume and pacemaker dependence at 30 days. A cutoff of $239.2 \mathrm{~mm}^{3}$ for calcium was used.

Fujita et al examined patients who received either balloonexpandable valves or self-expanding valves and found that left coronary cusp (LCC) leaflet calcification as well as DLZ calcification was significantly associated with the need for new pacemaker implantation after TAVR [24]. In a separate study, Maeno et al found that calcification in the NCC portion of the DLZ, as well as LCC and RCC leaflet calcification, was predictive of need for new PPM implantation after TAVR with third-generation balloon-expandable valves [7]. These findings suggest that aortic valve complex calcification does play a role in conduction abnormalities after TAVR, though it is unclear exactly what distribution portends the highest risk. Furthermore, it is unclear whether aortic valve complex calcification or the specific distribution of calcification plays a role in long-term conduction abnormalities after TAVR.

Prior to the routine use of CT scans in TAVR pre-procedure planning, 3D transesophageal echocardiography (TEE) was a frequently used imaging modality to further visualize the aortic valve complex. NCC thickness was found to be an important predictor of PPM implantation after TAVR with selfexpanding valves [25]. The NCC lies in close proximity to the conduction system, as the left bundle courses in the interleaflet region between the NCC and RCC with the AV node close by $[25,26]$. In fact, this relationship between the NCC and AV node has been used during catheter ablation of the AV node $[27,28]$. Because the native aortic valve is not removed during TAVR but merely excluded, increased pressure on this interleaflet region during balloon expansion of the bioprosthetic valve expansion can lead to damage to the conduction system, which may be exacerbated by significant calcification of the adjacent NCC.

Interestingly, while NCC calcium volume was a strong predictor of eventual conduction system recovery, there was no significant difference in NCC calcium volume between patients with early and late recovery of their conduction system (Supplementary Material 3, www.cardiologyres.org). Furthermore, demographic, electrocardiographic, imaging and procedural parameters were not significantly different between these groups either (Supplementary Material 2, www.cardiologyres. org). This likely reflects the fact that conduction disease after TAVR is influenced by a multitude of factors, including preexisting conduction disease, intra-procedural complications, anatomy, sizing and calcification. However, given the small sample size of this study, a multivariate analysis for these subgroups would not be statistically valid and was not performed. Future studies with larger sample sizes may help clarify this issue further.

Because conduction abnormalities after TAVR often resolve over time, it is of particular interest to determine which patients will recover conduction and which patients will continue to have conduction abnormalities after TAVR. Interestingly, in our study, intra-procedural heart block was the only significant difference between patients who did not require PPM implantation after TAVR and those who required PPM implantation but were not pacemaker-dependent at 30 days. In contrast, patients who were pacemaker-dependent at 30 days had significantly higher rates of RBBB, intra-procedural heart block, bifascicular block and QRS duration than those who did not require PPM implantation (Table 2). One possible explanation for the similarity of patients who do not require PPM after TAVR and those who require PPM but are not pacemaker-dependent at 30 days is that the acute need for pacemaker in these patients is related to procedural related factors such as edema and mechanical injury. In contrast, patients who are pacemaker-dependent at 30 days have greater pre-existing conduction disease.

As with any other invasive procedure, PPM implantation 
is not benign, and PPM removal after implantation is not recommended solely because of recovery of conduction abnormalities [29, 30]. Furthermore, multiple studies have shown that long-term RV pacing is associated with worsening ejection fraction and congestive heart failure due to the loss of ventricular synchrony, which leads to increased morbidity and mortality [31-35]. PPM after TAVR has been associated with increased repeat hospitalizations, increased morbidity and mortality and decrease in left ventricular ejection fraction $[15$, $16,36]$.

At the same time, while conduction abnormalities after TAVR may be temporary in many cases, it is unlikely that patients will be kept in the hospital long enough to allow complete recovery of the conduction system and avoid PPM implantation. However, identification of patients at high or low risk of permanent conduction abnormalities would be helpful in determining the optimal pacing strategies for these patients. For example, patients with a low risk of permanent conduction abnormalities may only require a singular ventricular lead for backup pacing, while patients with a high risk of permanent abnormalities would warrant more aggressive device implantation and pacing strategies.

\section{Limitations}

The major limitations of this study are the mono-centric study design as well as the relatively small sample size. The rate of pacemaker implantation in this study $(11.1 \%)$ is slightly higher than that reported in other large studies of balloon-expandable valve implantation (8.8\%) [15]. The exact intra-procedural implant depth, which has been associated with pacemaker implantation in a few studies, was not reported at the time of the procedure and not analyzed in this study $[37,38]$. Furthermore, although rates of pacemaker implantation vary slightly across different generations of the SAPIEN valve, predictors were not analyzed separately based on valve generation in this study because of the small sample size. Clinical outcomes, such as mortality, LVEF and re-hospitalization were not assessed in this study. Finally, aortic valve calcification was not quantified at the time of the study and was analyzed retrospectively. Future multicenter studies will help to create prediction models to identify patients at risk of long-term conduction abnormalities after TAVR.

\section{Conclusion}

In this single-center study, conduction disturbances after TAVR with a balloon-expandable valve requiring pacemaker implantation recovered in the majority of patients $(68 \%)$ at 30 days post-PPM implantation. NCC leaflet calcification as quantified by pre-TAVR CT was found to be a novel and powerful predictor of pacemaker dependence after TAVR. As indications for TAVR expand to younger and lower risk patients, it will become increasingly important to develop accurate prediction models for conduction abnormalities after TAVR to determine optimal pacing strategies in this growing patient population.

\section{Supplementary Material}

Suppl 1. Comparison of Calcium Volume Distribution by Leaflet.

Suppl 2. Demographic, Electrocardiographic, Echocardiographic and Procedural Parameters of Non-RV Pacing Patients at 10 Days Post-PPM Implant vs. Non-RV Pacing and Pacing Dependent Patients at 1 Month Post-PPM Implant.

Suppl 3. A. Aortic Valve Complex Calcification in Patients With Early and Late Recovery of Conduction. B. Aortic Valve Complex Calcification in Patients With Early Recovery of Conduction and No Recovery of Conduction. C. Aortic Valve Complex Calcification in Patients With Late Recovery of Conduction and No Recovery of Conduction.

\section{Acknowledgments}

None to declare.

\section{Financial Disclosure}

None to declare.

\section{Conflict of Interest}

None to declare.

\section{Informed Consent}

Not applicable.

\section{Author Contributions}

ES: data collection, data analysis, manuscript preparation; BM: data collection; DG: manuscript preparation; MA: data collection, data analysis; SC: data collection, data analysis; AP: data collection; WS: data collection; MA: data collection; $\mathrm{AC}$ : data collection, data analysis, manuscript preparation.

\section{Data Availability}

The authors declare that data supporting the findings of this study are available within the article.

\section{References}

1. Khatri PJ, Webb JG, Rodes-Cabau J, Fremes SE, Ruel M, Lau K, Guo H, et al. Adverse effects associated with transcatheter aortic valve implantation: a meta-analysis of contemporary studies. Ann Intern Med. 2013;158(1):35- 
46.

2. Jilaihawi H, Chakravarty T, Weiss RE, Fontana GP, Forrester J, Makkar RR. Meta-analysis of complications in aortic valve replacement: comparison of MedtronicCorevalve, Edwards-Sapien and surgical aortic valve replacement in 8,536 patients. Catheter Cardiovasc Interv. 2012;80(1):128-138.

3. Nazif TM, Williams MR, Hahn RT, Kapadia S, Babaliaros V, Rodes-Cabau J, Szeto WY, et al. Clinical implications of new-onset left bundle branch block after transcatheter aortic valve replacement: analysis of the PARTNER experience. Eur Heart J. 2014;35(24):1599-1607.

4. Erkapic D, De Rosa S, Kelava A, Lehmann R, Fichtlscherer S, Hohnloser SH. Risk for permanent pacemaker after transcatheter aortic valve implantation: a comprehensive analysis of the literature. J Cardiovasc Electrophysiol. 2012;23(4):391-397.

5. Maan A, Refaat MM, Heist EK, Passeri J, Inglessis I, Ptaszek L, Vlahakes G, et al. Incidence and predictors of pacemaker implantation in patients undergoing transcatheter aortic valve replacement. Pacing Clin Electrophysiol. 2015;38(7):878-886.

6. Auffret V, Puri R, Urena M, Chamandi C, Rodriguez-Gabella T, Philippon F, Rodes-Cabau J. Conduction disturbances after transcatheter aortic valve replacement: current status and future perspectives. Circulation. 2017;136(11):10491069.

7. Maeno Y, Abramowitz Y, Kawamori H, Kazuno Y, Kubo $\mathrm{S}$, Takahashi N, Mangat G, et al. A highly predictive risk model for pacemaker implantation after TAVR. JACC Cardiovasc Imaging. 2017;10(10 Pt A):1139-1147.

8. Kawashima T, Sato F. Visualizing anatomical evidences on atrioventricular conduction system for TAVI. Int J Cardiol. 2014;174(1):1-6.

9. Young Lee M, Chilakamarri Yeshwant S, Chava S, Lawrence Lustgarten D. Mechanisms of heart block after transcatheter aortic valve replacement - cardiac anatomy, clinical predictors and mechanical factors that contribute to permanent pacemaker implantation. Arrhythm Electrophysiol Rev. 2015;4(2):81-85.

10. Schernthaner C, Kraus J, Danmayr F, Hammerer M, Schneider J, Hoppe UC, Strohmer B. Short-term pacemaker dependency after transcatheter aortic valve implantation. Wien Klin Wochenschr. 2016;128(5-6):198-203.

11. Simms AD, Hogarth AJ, Hudson EA, Worsnop VL, Blackman DJ, O'Regan DJ, Tayebjee MH. Ongoing requirement for pacing post-transcatheter aortic valve implantation and surgical aortic valve replacement. Interact Cardiovasc Thorac Surg. 2013;17(2):328-333.

12. van der Boon RM, Van Mieghem NM, Theuns DA, Nuis RJ, Nauta ST, Serruys PW, Jordaens L, et al. Pacemaker dependency after transcatheter aortic valve implantation with the self-expanding Medtronic CoreValve System. Int J Cardiol. 2013;168(2):1269-1273.

13. Sharma E, Chu AF. Predictors of right ventricular pacing and pacemaker dependence in transcatheter aortic valve replacement patients. J Interv Card Electrophysiol. 2018;51(1):77-86.

14. Chevreul K, Brunn M, Cadier B, Haour G, Eltchaninoff
H, Prat A, Leguerrier A, et al. Cost of transcatheter aortic valve implantation and factors associated with higher hospital stay cost in patients of the FRANCE (FRench Aortic National CoreValve and Edwards) registry. Arch Cardiovasc Dis. 2013;106(4):209-219.

15. Nazif TM, Dizon JM, Hahn RT, Xu K, Babaliaros V, Douglas PS, El-Chami MF, et al. Predictors and clinical outcomes of permanent pacemaker implantation after transcatheter aortic valve replacement: the PARTNER (Placement of AoRtic TraNscathetER Valves) trial and registry. JACC Cardiovasc Interv. 2015;8(1 Pt A):60-69.

16. Urena M, Webb JG, Tamburino C, Munoz-Garcia AJ, Cheema A, Dager AE, Serra V, et al. Permanent pacemaker implantation after transcatheter aortic valve implantation: impact on late clinical outcomes and left ventricular function. Circulation. 2014;129(11):1233-1243.

17. Nagatomo T, Abe H, Kikuchi K, Nakashima Y. New onset of pacemaker dependency after permanent pacemaker implantation. Pacing Clin Electrophysiol. 2004;27(4):475479.

18. Khalique OK, Hahn RT, Gada H, Nazif TM, Vahl TP, George I, Kalesan B, et al. Quantity and location of aortic valve complex calcification predicts severity and location of paravalvular regurgitation and frequency of post-dilation after balloon-expandable transcatheter aortic valve replacement. JACC Cardiovasc Interv. 2014;7(8):885894.

19. Ewe SH, Ng AC, Schuijf JD, van der Kley F, Colli A, Palmen M, de Weger A, et al. Location and severity of aortic valve calcium and implications for aortic regurgitation after transcatheter aortic valve implantation. Am J Cardiol. 2011;108(10):1470-1477.

20. Schultz CJ, Tzikas A, Moelker A, Rossi A, Nuis RJ, Geleijnse MM, van Mieghem N, et al. Correlates on MSCT of paravalvular aortic regurgitation after transcatheter aortic valve implantation using the Medtronic CoreValve prosthesis. Catheter Cardiovasc Interv. 2011;78(3):446-455

21. Callister TQ, Cooil B, Raya SP, Lippolis NJ, Russo DJ, Raggi P. Coronary artery disease: improved reproducibility of calcium scoring with an electron-beam CT volumetric method. Radiology. 1998;208(3):807-814.

22. Glodny B, Helmel B, Trieb T, Schenk C, Taferner B, Unterholzner V, Strasak A, et al. A method for calcium quantification by means of CT coronary angiography using 64-multidetector CT: very high correlation with Agatston and volume scores. Eur Radiol. 2009;19(7):1661-1668.

23. Cerillo AG, Mariani M, Berti S, Glauber M. Sizing the aortic annulus. Ann Cardiothorac Surg. 2012;1(2):245-256.

24. Fujita B, Kutting M, Seiffert M, Scholtz S, Egron S, Prashovikj E, Borgermann J, et al. Calcium distribution patterns of the aortic valve as a risk factor for the need of permanent pacemaker implantation after transcatheter aortic valve implantation. Eur Heart J Cardiovasc Imaging. 2016;17(12):1385-1393.

25. Jilaihawi H, Chin D, Vasa-Nicotera M, Jeilan M, Spyt T, $\mathrm{Ng}$ GA, Bence J, et al. Predictors for permanent pacemaker requirement after transcatheter aortic valve implantation with the CoreValve bioprosthesis. Am Heart J. 
2009;157(5):860-866.

26. Piazza N, de Jaegere P, Schultz C, Becker AE, Serruys $\mathrm{PW}$, Anderson RH. Anatomy of the aortic valvar complex and its implications for transcatheter implantation of the aortic valve. Circ Cardiovasc Interv. 2008;1(1):74-81.

27. Das S, Neuzil P, Albert CM, D'Avila A, Mansour M, Mela T, Ellinor PT, et al. Catheter ablation of peri-AV nodal atrial tachycardia from the noncoronary cusp of the aortic valve. J Cardiovasc Electrophysiol. 2008;19(3):231-237.

28. Tada H, Naito S, Miyazaki A, Oshima S, Nogami A, Taniguchi K. Successful catheter ablation of atrial tachycardia originating near the atrioventricular node from the noncoronary sinus of Valsalva. Pacing Clin Electrophysiol. 2004;27(10):1440-1443.

29. Kotsakou M, Kioumis I, Lazaridis G, Pitsiou G, Lampaki S, Papaiwannou A, Karavergou A, et al. Pacemaker insertion. Ann Transl Med. 2015;3(3):42.

30. Kusumoto FM, Schoenfeld MH, Wilkoff BL, Berul CI, Birgersdotter-Green UM, Carrillo R, Cha YM, et al. 2017 HRS expert consensus statement on cardiovascular implantable electronic device lead management and extraction. Heart Rhythm. 2017;14(12):e503-e551.

31. Lamas GA, Lee KL, Sweeney MO, Silverman R, Leon A, Yee R, Marinchak RA, et al. Ventricular pacing or dual-chamber pacing for sinus-node dysfunction. N Engl J Med. 2002;346(24):1854-1862.

32. Sweeney MO, Hellkamp AS, Ellenbogen KA, Greenspon AJ, Freedman RA, Lee KL, Lamas GA, et al. Adverse effect of ventricular pacing on heart failure and atrial fibrillation among patients with normal baseline QRS duration in a clinical trial of pacemaker therapy for sinus node dysfunction. Circulation. 2003;107(23):2932-2937.

33. De Sisti A, Marquez MF, Tonet J, Bonny A, Frank R, Hidden-Lucet F. Adverse effects of long-term right ventricular apical pacing and identification of patients at risk of atrial fibrillation and heart failure. Pacing Clin Electrophysiol. 2012;35(8):1035-1043.

34. Tops LF, Schalij MJ, Bax JJ. The effects of right ventricular apical pacing on ventricular function and dyssynchrony implications for therapy. J Am Coll Cardiol. 2009;54(9):764-776.

35. Wilkoff BL, Cook JR, Epstein AE, Greene HL, Hallstrom AP, Hsia H, Kutalek SP, et al. Dual-chamber pacing or ventricular backup pacing in patients with an implantable defibrillator: the Dual Chamber and VVI Implantable Defibrillator (DAVID) Trial. JAMA. 2002;288(24):31153123.

36. Aljabbary T, Qiu F, Masih S, Fang J, Elbaz-Greener G, Austin PC, Rodes-Cabau J, et al. Association of clinical and economic outcomes with permanent pacemaker implantation after transcatheter aortic valve replacement. JAMA Netw Open. 2018;1(1):e180088.

37. Binder RK, Webb JG, Toggweiler S, Freeman M, Barbanti $\mathrm{M}$, Willson AB, Alhassan D, et al. Impact of post-implant SAPIEN XT geometry and position on conduction disturbances, hemodynamic performance, and paravalvular regurgitation. JACC Cardiovasc Interv. 2013;6(5):462-468.

38. Mauri V, Reimann A, Stern D, Scherner M, Kuhn E, Rudolph V, Rosenkranz S, et al. Predictors of permanent pacemaker implantation after transcatheter aortic valve replacement with the SAPIEN 3. JACC Cardiovasc Interv. 2016;9(21):2200-2209. 